\title{
The Reaction of Cyanoacetylhydrazine with Chloroacetone: Synthesis of 1,2,4-Triazine, 1,3,4-Oxadiazine and Their Fused Derivatives with Antitumor Activities
}

\author{
Rafat M. Mohareb ${ }^{1,2^{*}}$, Eman M. Samir ${ }^{3}$ \\ ${ }^{1}$ Department of Organic Chemistry, Faculty of Pharmacy, October University for Modern Sciences \& Arts, Giza, Egypt \\ ${ }^{2}$ Department of Chemistry, Faculty of Science, Cairo University, Giza, Egypt \\ ${ }^{3}$ National Organization for Drug Control \& Research, Cairo, Egypt \\ Email: 'raafat_mohareb@yahoo.com
}

Received January 17, 2012; revised February 15, 2012; accepted February 25, 2012

\begin{abstract}
The reaction of cyanoacetylhydrazine with chloroacetone gave the N-(1-chloropropan-2-ylidene)-2-cyanoacetohydrazide. This compound reacted with either hydrazine hydrate or phenylhydrazine to give the corresponding 1,2,4-triazine derivatives. On the other hand, its reaction with either benzenediazonium chloride or benzaldehyde gave in each case the 1,3,4-oxadiazine derivatives. Moreover, the reaction of the cyanoacetylhydrazine with 2-boromocyclohexanone gave the corresponding hydrazine-hydrozon derivative. The antitumor evaluation of the newly synthesized products against three cancer cell lines, namely breast adenocarcinoma (MCF-7), non-small cell lung cancer (NCI-H460) and CNS cancer (SF-268) was recorded. Some of the tested compounds showed activities which was higher than the reference doxorubicin.
\end{abstract}

Keywords: 1,2,4-Triazine; 1,3,4-Oxadiazine; Pyrazole; Antitumor

\section{Introduction}

It is well known that the hydrazone group plays an important role for the antimicrobial activity. Furthermore, a number of hydrazide-hydrazone claimed to possess interesting antibacterial antifungal [1-3] anticonvulsant [4-6] anti-inflammatory [7-9] antimalarial [10] and antituberculosis activities [11-15] With the aim of obtaining new hydrazide-hydrazones with such wide spectrum of pharamaceutical applications, we report here the synthesis of a series of hydrazide-hydrzones via the reaction of cyanoacetylhydrazine (1) with $\alpha$-haloketones $[16,17]$. All synthesized compounds have been screened for antitumor activity against breast adenocarcinoma (MCF-7), non-small cell lung cancer (NCI-H460) and CNS cancer (SF-268).

\section{Results and Discussions}

The reaction of $\mathbf{1}$ with chloroacetone (2) in 1,4-dioxane gave the hydrazide-hydrazon derivative 3 . The structure of compound 3 was based on the analytical and spectral data. Thus, the ${ }^{1} \mathrm{H}$ NMR spectrum of the reaction product showed the presence of a singlet at $\delta 2.36 \mathrm{ppm}$ corresponding to $\mathrm{CH}_{3}$ group, two singlets at $\delta 3.37,4.02 \mathrm{ppm}$

${ }^{*}$ Corresponding author. corresponding to two $\mathrm{CH}_{2}$ groups and a singlet at $\delta 8.09$ ppm for the NH group. Further confirmation for the structure of compound $\mathbf{3}$ was obtained through studying its reactivity towards some chemical reagents. Thus, the reaction of $\mathbf{3}$ with either hydrazine hydrate (4a) or phenylhydrazine (4b) gave the 1,2,4 triazine derivatives $\mathbf{6 a}$ and $\mathbf{6 b}$, respectively. Formation of the latter products is explained through the intermediate formation of $\mathbf{5 a}, \mathbf{b}$ followed by water elimination.

The reaction of $\mathbf{6 b}$ with benzenediazonium chloride (7) gave the phenyl hydrazone derivative 9 (Scheme 1). The reaction took place through the first coupling of $\mathbf{6}$ with $\mathbf{7}$ to give the phenylhydrazone derivative 8 followed by Micheal addition of the N-NH group present in the triazine ring to the cyano group to give the pyrazolo[5-1-c] $[1,2,4]$ triazine derivative $\mathbf{9}$. On the other hand, the reaction of $\mathbf{6 b}$ with either malononitrile (10a) or ethyl cyanoacetate (10b) gave the tricyclic products 11a and 11b, respectively. Analytical and spectral data of 11a,b are consistent with the proposed structures (see experimental section). The reaction of compound 3 with either benzenediazonium chloride 7 or p-chlorobenzenediazonium chloride $\mathbf{1 2}$ gave 1,3,4-oxadiazine derivatives 13a and $13 \mathbf{b}$ respectively. On the other hand, the reaction of compound 3 with potassium cyanide (14) gave the 3 -amino 

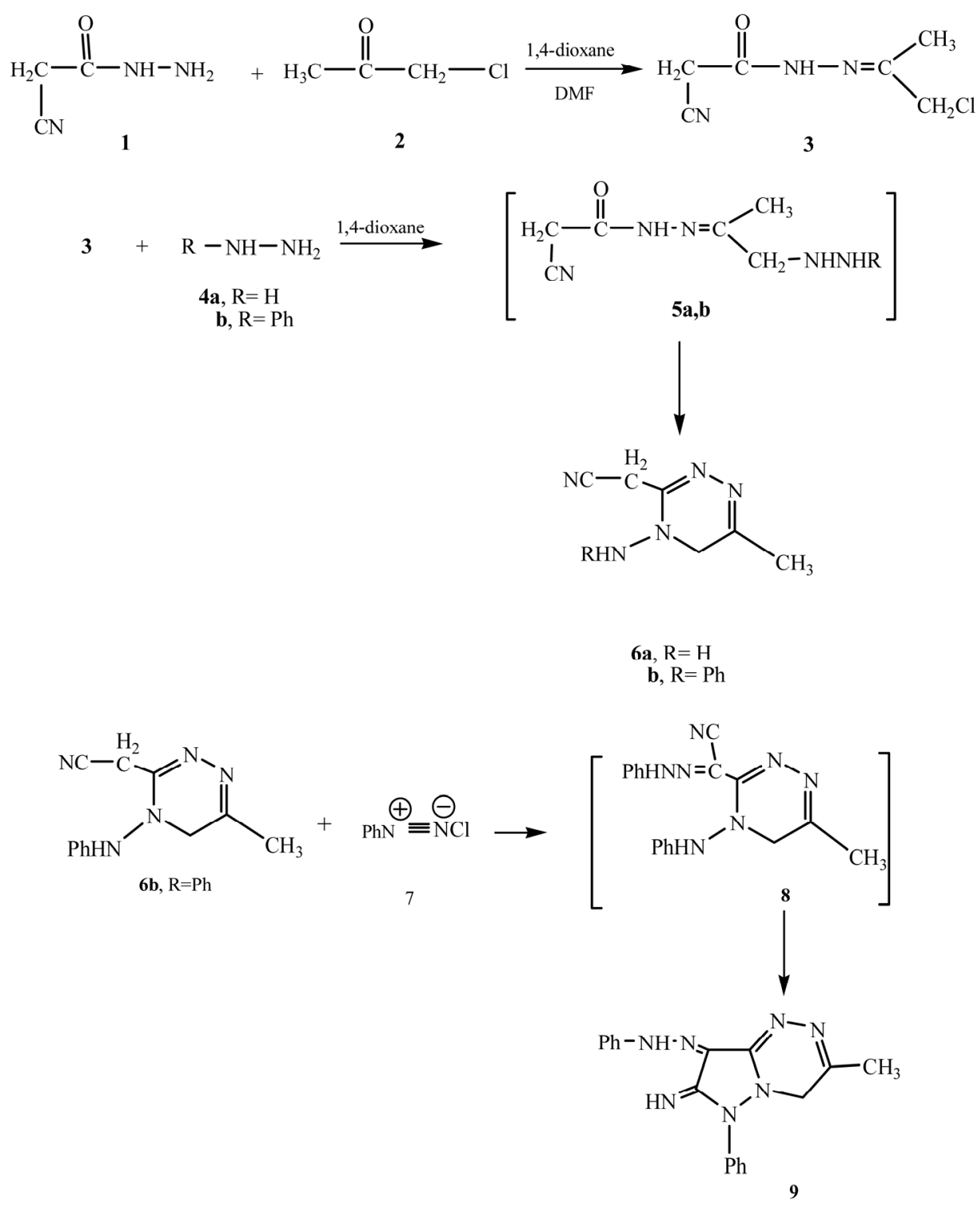

Scheme 1. Synthesis of compounds 3 - 9.

pyrazole derivative 15. Formation of the pyrazole derivative $\mathbf{1 5}$ took place in analogous with our recent reported work [18].

The reaction of compound 3 with benzaldehyde (16) gave the 2- $\alpha$-benzal acetonitryl-1,3,4-oxadiazine derivatives 18 (Scheme 2) its formation occured through the first formation of the benzal derivative followed by $\mathrm{HCl}$ liberation. The ${ }^{1}$ HNMR spectrum of the latter product showed the presence of a singlet at $\delta 2.68$ for $\mathrm{CH}_{3}$ group, a singlet at $\delta 5.26$ for oxadiazine $\mathrm{CH}_{2}$ group, a singlet at $\delta 6.05$ for $\mathrm{CH}=\mathrm{C}$ and a multiplet at $\delta 7.22$ 7.35 for $\mathrm{C}_{6} \mathrm{H}_{5}$ group.

The reaction of compound 18 with either malononitrile (10a) or ethyl cyanoacetate (10b) gave the pyrido [2,1-b] 1,3,4-oxadiazine derivatives 20a and 20b. The structure elucidation of the latter products was based on analytical and spectral data (see experimental section). Next, we studied the reaction of cyanoacetylhydrazine (1) with 2bromocyclohexanone (21) in 1,4-dioxane at room temperature. The reaction leads to the formation of the hydrazide-hydrazone derivative 22 . The analytical and spectral and spectral data of the latter product are in agreement with the proposed structure (see experimental section).

Compound 22 reacted with either hydrazine hydrate (4a) or phenylhydrazine (4b) to give the tricyclic products 24a and 24b, respectively. The formation of 24a,b was explained in terms of intermediate formation of hydrazono derivatives followed by cyclization to give the intermediates 23a,b, respectively. The latter intermediates underwent further cyclization to give 24a,b. The reaction of $\mathbf{2 2}$ with either benzenediazonium chloride (7) 
or p-chlorobenzenediazonium chloride (12) gave the $\alpha$-phenyl hydrazone acetonitrilo-tetrahydrobenzo $[e]-1,3,4$ oxadiazine derivatives 25a,b. The analytical and spectral data the latter products are in agreement with the proposed structure (Scheme 3).

\section{Antitumor Activity}

\subsection{Material and Methods \& Reagents}

Fetal bovine serum (FBS) and L-glutamine, were from Gibco Invitrogen Co. (Scotland, UK). RPMI-1640 medium was from Cambrex (New Jersey, USA). Dimethyl sulfoxide (DMSO), doxorubicin, penicillin, streptomycin and sulforhodamine B (SRB) were from Sigma Chemical Co. (Saint Louis, USA). Samples: Stock solutions of new compounds through $\mathbf{3}$ - 25b were prepared in DMSO and kept at $-20^{\circ} \mathrm{C}$. Appropriate dilutions of the compounds were freshly prepared just prior the assays. Final concentrations of DMSO did not interfere with the cell growth.

Cell cultures. Three human tumor cell lines, MCF-7 (breast adenocarcinoma), NCI-H460 (non-small cell lung cancer), and SF-268 (CNS cancer) were used. MCF-7 was obtained from the European Collection of Cell Cultures (ECACC, Salisbury, UK) and NCI-H460 and SF-268 were<smiles>[X]CC#N</smiles><smiles>[Y]C1=CC(N)=C2C3=NN=C(C)CN3N(c3ccccc3)C2N1</smiles>

11a, $\mathrm{Y}=\mathrm{NH}_{2}$ b, $\mathrm{Y}=\mathrm{OH}$

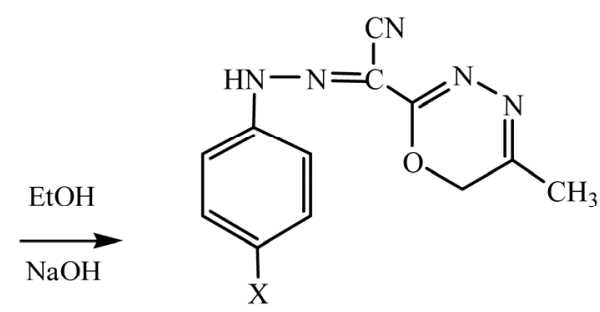

$$
\begin{aligned}
& \text { 7, } \mathrm{X}=\mathrm{H} \\
& \mathbf{1 2}, \mathrm{X}=\mathrm{Cl}
\end{aligned}
$$

13a, $\mathrm{X}=\mathrm{H}$

b, $\mathrm{X}=\mathrm{Cl}$

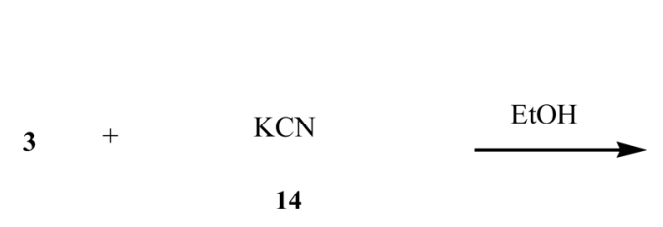<smiles>Cc1cc(N)n(C(=O)[CH]C#N)n1</smiles>

15<smiles>CC1=NN=C(/C(C#N)=C/[In])OC1</smiles>

Scheme 2. Synthesis of compounds 11a,b-18. 


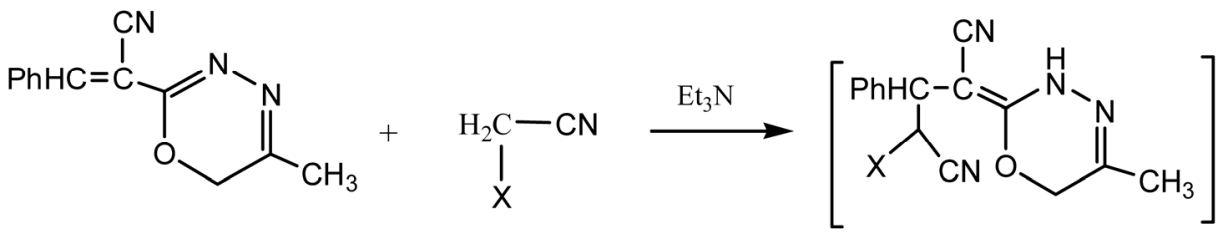

18

10a, $\mathrm{X}=\mathrm{CN}$

b, $\mathrm{X}=\mathrm{COOEt}$

19<smiles>N#CCC(=O)NN</smiles><smiles>O=C1CCCCC1Br</smiles><smiles>[Y]C1=C(C#N)C(c2ccccc2)C(C#N)=C2OCC(C)=NN12</smiles>

21

$$
\begin{aligned}
& \mathbf{2 0 a}, \mathrm{Y}=\mathrm{NH}_{2} \\
& \mathbf{b}, \mathrm{Y}-\mathrm{OH}
\end{aligned}
$$<smiles>N#CCC(=O)N=C1CCCCC1Br</smiles>

22

$$
\begin{gathered}
\text { 4a, } \mathrm{R}=\mathrm{H} \\
\mathbf{b}, \mathrm{R}=\mathrm{Ph}
\end{gathered}
$$<smiles>[GeH3]</smiles><smiles>[R]NN1C(C[AlH2])=NN=C2CCCCC21</smiles><smiles></smiles>

24a, $\mathrm{R}=\mathrm{H}$ b, $\mathrm{R}=\mathrm{Ph}$<smiles>[Y10][Y16]([Y16])([H])Cl</smiles>

Scheme 3. Synthesis of compounds 20a,b - 25a,b.

kindly provided by the National Cancer Institute (NCI, Cairo, Egypt). They grow as monolayer and rou- tinely maintained in RPMI-1640 medium supplemented with $5 \%$ heat inactivated FBS, $2 \mathrm{mM}$ glutamine and antibiotics (penicillin $100 \mathrm{U} / \mathrm{mL}$, streptomycin $100 \mathrm{~g} / \mathrm{mL}$ ), at $37^{\circ} \mathrm{C}$ in a humidified atmosphere containing $5 \% \mathrm{CO}_{2}$.
Exponentially growing cells were obtained by plating 1.5 $\times 10^{5}$ cells $/ \mathrm{mL}$ for MCF-7 and SF-268 and $0.75 \times 10^{4}$ cells $/ \mathrm{mL}$ for NCI-H460, followed by $24 \mathrm{~h}$ of incubation. The effect of the vehicle solvent (DMSO) on the growth of these cell lines was evaluated in all the experiments by exposing untreated control cells to the maximum con- 
centration $(0.5 \%)$ of DMSO used in each assay.

\subsection{Effect on the Growth of Human Tumor Cell Lines}

The effect of compounds $\mathbf{3}$ - 25b was evaluated on the in vitro growth of three human tumor cell lines representing different tumor types, namely, breast adenocarcinoma (MCF-7), non-small cell lung cancer (NCI-H460) and CNS cancer (SF-268), after a continuous exposure of 48 h. The results are summarized in Table 1. All the compounds were able to inhibit the growth of the human tumor cell lines in a dose dependent manner (data not shown). Compound 11a (7-methyl-9-phenyl-8,9-dihdro-1, 5,6,8a,9-pentaaza-fluorene-2,4-diamine) and 20b (2,8dihydro-6-hydroxy-3-methyl-8-phenylpyrido[2,1-b][1,3, 4] oxadiazine-7,9-dicarbonitrile) showed the best results, exhibiting an equivalent potency in all the three tumor cell lines; these values are much lower than that of the gram positive control doxorubicin. Compounds 20b and $24 \mathbf{b}$ are the following in relation to the anticancer activity. On the other hand, compounds 3, 6a, 9, 11a, 18, 20a, 22, 24a and 25b showed moderated growth inhibitory effect which showed $\mathrm{GI}_{50}<30 \mathrm{~mol} \cdot \mathrm{L}^{-1}$.

Comparing the activities of 20a and 20b it is observed that the 2-amino group in 20a showed a stronger growth inhibitory effect than the 2-hydroxy substituent in 20b although the results in NCI-H460 cell line are comparable. Also comparing compounds $\mathbf{2 4 a}$ and $\mathbf{2 4 b}$, it is obvious

Table 1. Effect of compounds 3 - 25b on the growth of three human tumor cell lines.

\begin{tabular}{llll}
\hline \multirow{2}{*}{ Compound } & \multicolumn{3}{c}{$\mathrm{GI}_{50}\left(\mu \mathrm{mol} \cdot \mathrm{L}^{-1}\right)$} \\
\cline { 2 - 4 } No. & MCF-7 & NCI-H460 & SF-268 \\
\hline $\mathbf{3}$ & $8.0 \pm 0.3$ & $12.6 \pm 1.8$ & $12.3 \pm 1.0$ \\
6a & $12.0 \pm 2.2$ & $22.3 \pm 3.6$ & $33 \pm 0.4$ \\
$\mathbf{6 b}$ & $66.2 \pm 12.7$ & $30.9 \pm 8.2$ & $42.8 \pm 8.3$ \\
$\mathbf{9}$ & $10.2 \pm 0.8$ & $8.6 \pm 1.4$ & $6.2 \pm 1.6$ \\
11a & $0.02 \pm 0.002$ & $0.03 \pm 0.01$ & $0.01 \pm 0.002$ \\
11b & $30.5+12.0$ & $20.1 \pm 12.8$ & $20.0 \pm 4.31$ \\
13a & $30.2 \pm 2.2$ & $22.2 \pm 6.2$ & $12.6 \pm 2.6$ \\
13b & $16.2 \pm 2.2$ & $20.6 \pm 1.6$ & $33.2 \pm 3.7$ \\
15 & $30.0 \pm 2.5$ & $22.0 \pm 4.6$ & $20.5 \pm 2.8$ \\
18 & $14.0 \pm 0.4$ & $12.3 \pm 0.6$ & $26.5 \pm 4.3$ \\
20a & $8.4 \pm 2.8$ & $14.1 \pm 0.6$ & $20.3 \pm 0.5$ \\
20b & $0.02 \pm 0.002$ & $0.01 \pm 0.008$ & $0.02 \pm 0.008$ \\
22 & $22.8 \pm 10.0$ & $30.2 \pm 6.4$ & $18.2 \pm 10.6$ \\
24a & $20.2 \pm 8.2$ & $16.0 \pm 2.6$ & $12.2 \pm 4.8$ \\
24b & $0.4 \pm 0.08$ & $0.2 \pm 0.04$ & $0.1 \pm 0.01$ \\
25a & $40.6 \pm 10.0$ & $32.6 \pm 8.0$ & $62.4 \pm 14.8$ \\
25b & $12.2 \pm 4.0$ & $16.0 \pm 2.8$ & $14.2 \pm 4.6$ \\
Doxorubicin & $0.04 \pm 0.008$ & $0.09 \pm 0.008$ & $0.09 \pm 0.007$ \\
\hline
\end{tabular}

Results are given in concentrations that were able to cause $50 \%$ of cell growth inhibition $\left(\mathrm{GI}_{50}\right)$ after a continuous exposure of $48 \mathrm{~h}$ and show means $\pm \mathrm{SEM}$ of three-independent experiments performed in duplicate. that compound 24b which is the (1-phenyl-1,6,7,8,9ahexahydro-benzo[e]pyrazolo[5,1-c][1,2,4]triazin-2-ylami ne) with its $\mathrm{N}-\mathrm{H}$ group is much higher that $\mathbf{2 4 b}$ with its $\mathrm{N}-\mathrm{Ph}$ group. It is obvious that compounds $\mathbf{6 b}, \mathbf{1 1 b}, \mathbf{1 3 a}$ and 25a showed the lowest inhibitory effect towards the three cell line. The relative inhibitory effect of the newly synthesized and the reference doxorubicin, towards the three cancer cell lines, is indicated through Figure 1.

\section{Experimental}

\section{Materials}

The requisite starting materials such as chloroacetone, hydrazine hydrate, phenyl hydrazine, sodium nitrite, malononitrile, ethyl cyanoacetate, aniline, p-chloro aniline, potassium cyanide, benzaldehyde, bromocyclohexanone were produced from Aldrich Company and used without any further purification. All the solvents were purified and dried by standard method. All melting points were determined in open capillaries and are uncorrected. IR spectra were measured using $\mathrm{KBr}$ discs on a Pye Unicam SP-1000 spectrophotometer. ${ }^{1} \mathrm{HNMR}$ spectra was measured on a varian EM390-200 MHz instrument in DMSO as solvent using tetramethylsilane (TMS) as internal reference standard. The chemical shifts are quoted as $\delta \mathrm{ppm}$.

\section{N-(1-Chloropropan-2-ylidene)-2-cyanoacetohydrazi} de (3).

Equimolecular amounts of $1(0.99 \mathrm{~g}, 0.01 \mathrm{~mol})$ and chloroacetone (2) $(0.92 \mathrm{~g}, 0.01 \mathrm{~mol})$ in 1,4-dioxane (25 $\mathrm{mL})$ and drops of dimethylformamide $(5 \mathrm{~mL})$ were stirred at room temperature for $1 \mathrm{~h}$. The formed solid product was collected by filtration. White crystals from ethanol in a yield $88 \%(1.52) \mathrm{g}$ and with m.p. $160^{\circ} \mathrm{C}$; Calculated for $\mathrm{C}_{6} \mathrm{H}_{8} \mathrm{~N}_{3} \mathrm{OCl}$ (173.59): C, 41.51; H, 4.64; $\mathrm{N}, 24.20$. Found: C, 40.89; H, 4.10; N, 23.75. IR $\left(\mathrm{V} / \mathrm{cm}^{-1}\right)$ $=3450-3329(\mathrm{NH}), 2892,2877\left(\mathrm{CH}_{3}, \mathrm{CH}_{2}\right), 2260(\mathrm{CN})$, $1688(\mathrm{C}=\mathrm{O}) .{ }^{1} \mathrm{HNMR}(\delta \mathrm{ppm}): 2.36\left(\mathrm{~s}, 3 \mathrm{H}, \mathrm{CH}_{3}\right), 3.37$,

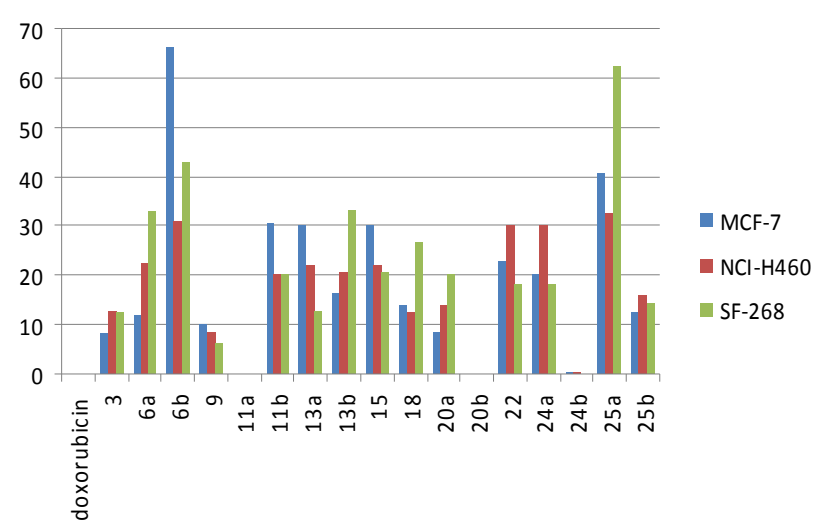

Figure 1. Inhibition of cell growth of the newly synthesized products towards the three cancer cell lines. 
$4.02\left(2 \mathrm{~s}, 4 \mathrm{H}, 2 \mathrm{CH}_{2}\right), 8.09$ (s, 1H, NH); MS: $m / z 173\left(\mathrm{M}^{+}\right.$, $10 \%)$.

3[(4-Amino-6-methyl-4,5-dihydro-[1,2,4]triazin-3-yl )]-acetonitrile (6a) and [6-Methyl-4-(N'-phenyl-hydra zino)-4,5-dihydro-[1,2,4]triazin-3-yl]-acetonitrile (6b).

General procedure: To a solution of 3 (1.73 g, 0.01 $\mathrm{mol})$ in 1,4-dioxane $(30 \mathrm{~mL})$, and either hydrazine hydrate (4a) $(0.50 \mathrm{~g}, 0.01 \mathrm{~mol})$ or phenyl hydrazine (4b) $(1.08 \mathrm{~g}, 0.01 \mathrm{~mol})$ were added. The reaction mixture was kept on ice bath for $10 \mathrm{~min}$. The solid product formed upon dilution with ice/water containing hydrochloric acid (till $\mathrm{pH}_{6}$ ), was collected by filtration.

Compound 6a: Brown crystals from ethanol, yield, $60 \%(0.9)$ g, m.p. $>300^{\circ}$ C. Calculated for $\mathrm{C}_{6} \mathrm{H}_{9} \mathrm{~N}_{5}$ (151.16): C, 47.67; H, 6.00; N, 46.33. Found: C, 47.00; H, 5.55; N, 45.86. IR $\left(\mathrm{V} / \mathrm{cm}^{-1}\right)=3466-3328\left(\mathrm{NH}_{2}, \mathrm{NH}\right)$, 3061 (CH aromatic), $2988\left(\mathrm{CH}_{3}\right), 2226(\mathrm{CN}), 1658$ $(\mathrm{C}=\mathrm{N}), 1643(\mathrm{C}=\mathrm{C}) .{ }^{1} \mathrm{HNMR}(\delta \mathrm{ppm}): 2.86\left(\mathrm{~s}, 3 \mathrm{H}, \mathrm{CH}_{3}\right)$, $4.23\left(\mathrm{~s}, 2 \mathrm{H}, \mathrm{CH}_{2}\right), 5.08\left(\mathrm{~s}, 2 \mathrm{H}, \mathrm{NH}_{2}\right), 5.21(\mathrm{~s}, 2 \mathrm{H}$, triazine $\left.\mathrm{CH}_{2}\right)$; $\mathrm{MS}: m / z 151\left(\mathrm{M}^{+}, 60 \%\right)$.

Compound 6b: Buff crystals from 1,4-dioxane, yield, $72 \%$ (1.63) g, m.p. $180^{\circ}$ C. Calculated for $\mathrm{C}_{12} \mathrm{H}_{13} \mathrm{~N}_{5}$ (227.26): C, 63.42; H, 5.70; N, 30.80. Found: C, 62.89; $\mathrm{H}, 4.88 ; \mathrm{N}, 29.99$. IR $\left(\mathrm{V} / \mathrm{cm}^{-1}\right)=3476-3331(\mathrm{NH}), 3055$ $\left(\mathrm{CH}\right.$ aromatic), $2982\left(\mathrm{CH}_{3}\right), 2261(\mathrm{CN}), 1655(\mathrm{C}=\mathrm{N})$, $1648(\mathrm{C}=\mathrm{C}) .{ }^{1} \mathrm{HNMR}(\delta \mathrm{ppm}): 2.83\left(\mathrm{~s}, 3 \mathrm{H}, \mathrm{CH}_{3}\right), 4.19$ (s, $\left.2 \mathrm{H}, \mathrm{CH}_{2}\right), 5.28\left(\mathrm{~s}, 2 \mathrm{H}\right.$, triazine $\left.\mathrm{CH}_{2}\right), 7.28-7.39(\mathrm{~m}, 5 \mathrm{H}$, $\left.\mathrm{C}_{6} \mathrm{H}_{5}\right), 8.78$ (s, $\left.1 \mathrm{H}, \mathrm{NH}\right)$; MS: $m / z 227\left(\mathbf{M}^{+}, 40 \%\right.$ ).

8-(2-Phenylhydrazono)-3-methyl-6-phenylpyrazolo[ 5,1-c][1,2,4]triazin-7 $(4 H, 6 H, 8 H)$-imine (9).

To a cold solution $\left(0^{\circ} \mathrm{C}-5^{\circ} \mathrm{C}\right)$ of $6 \mathbf{b}(2.27 \mathrm{~g}, 0.01 \mathrm{~mol})$ in ethanol $(30 \mathrm{~mL})$ containing sodium hydroxide $(10 \%, 6$ $\mathrm{mL})$ benzenediazonium chloride $(7)(0.01 \mathrm{~mol})$ [obtained via the addition of sodium nitrite solution $(0.70 \mathrm{~g}, 0.01$ mol) to a cold solution $\left(0^{\circ} \mathrm{C}-5^{\circ} \mathrm{C}\right)$ of aniline $(0.93 \mathrm{~g}$, $0.01 \mathrm{~mol})$ in hydrochloric acid $(8 \mathrm{~mL})$ was added with continuous stirring ] was added with continuous stirring. The reaction mixture was stirred for an additional $2 \mathrm{~h}$ then the formed solid product was collected by filtration. Orange crystals from ethanol in a yield, $80 \%,(2.65) \mathrm{g}$, with m.p. $236^{\circ} \mathrm{C}-240^{\circ} \mathrm{C}$. Calculated for $\mathrm{C}_{18} \mathrm{H}_{17} \mathrm{~N}_{7}$ (331.15): C, 65.22; H, 5.13; N, 29.59, Found: C, 64.66; $\mathrm{H}, 4.76 ; \mathrm{N}, 28.91$. IR $\left(\mathrm{U} / \mathrm{cm}^{-1}\right)=3455-3323(2 \mathrm{NH})$, $3052\left(\mathrm{CH}\right.$ aromatic), $2980\left(\mathrm{CH}_{3}\right), 1655(\mathrm{C}=\mathrm{N}), 1648(\mathrm{C}=$ C). ${ }^{1} \mathrm{HNMR}(\delta \mathrm{ppm}): 2.83\left(\mathrm{~s}, 3 \mathrm{H}, \mathrm{CH}_{3}\right), 4.65(\mathrm{~s}, 1 \mathrm{H}$, $\mathrm{D}_{2}$ Oexchangeable, $\left.\mathrm{NH}\right), 5.26\left(\mathrm{~s}, 2 \mathrm{H}\right.$, triazine $\left.\mathrm{CH}_{2}\right), 7.29$ $7.37\left(\mathrm{~m}, 10 \mathrm{H}, 2 \mathrm{C}_{6} \mathrm{H}_{5}\right), 8.82\left(\mathrm{~s}, 1 \mathrm{H}, \mathrm{D}_{2} \mathrm{Oexchangeable}\right.$, $\mathrm{NH})$; MS: $m / z 331\left(\mathrm{M}^{+}, 100 \%\right)$.

7-Methyl-9-phenyl-8,9-dihydro-1,5,6,8a,9-pentaazafluorene-2,4-diamine (11a) and 4-Amino-7-methyl-9phenyl-8,9-dihydro-1,5,6,8a,9-pentaaza-fluoren-2-ol (11b).

General procedure: Equimolecular amounts of $\mathbf{6 b}$ $(2.27 \mathrm{~g}, 0.01 \mathrm{~mol})$ and either malononitrile (10a) $(0.66 \mathrm{~g}$,
$0.01 \mathrm{~mol}$ ) or ethyl cyanoacetate (10b) (1.13 g, $0.01 \mathrm{~mol})$ were added in 1,4-dioxane $(25 \mathrm{~mL})$ containing triethylamine $(1.00 \mathrm{~mL})$ were heated under reflux for $4 \mathrm{~h}$. The formed solid product upon dilution with ice/water containing hydrochloric acid (till $\mathrm{pH}_{6}$ ), was collected by filtration.

Compound (11a): Red crystals from ethanol, yield $76 \%$ (2.22) g, m.p. $160^{\circ}$ C. Calculated for $\mathrm{C}_{15} \mathrm{H}_{17} \mathrm{~N}_{7}$ (293.33) C, 61.00; H, 5.80; N, 33.20. Found: C, 61.27; H, 5.53; N,33.44. IR $\left(\mathrm{U} / \mathrm{cm}^{-1}\right)=3473-3344\left(2 \mathrm{NH}_{2}\right), 3057$ $(\mathrm{CH}$ aromatic $), 2980\left(\mathrm{CH}_{3}\right), 1662(\mathrm{C}=\mathrm{N}), 1640(\mathrm{C}=$ C). ${ }^{1} \mathrm{HNMR}(\delta \mathrm{ppm}): 2.79\left(\mathrm{~s}, 3 \mathrm{H}, \mathrm{CH}_{3}\right), 4.43,5.18(2 \mathrm{~s}$, $\left.4 \mathrm{H}, 2 \mathrm{NH}_{2}\right), 5.20\left(\mathrm{~s}, 2 \mathrm{H}\right.$, triazine $\left.\mathrm{CH}_{2}\right), 7.23-7.36(\mathrm{~m}, 8 \mathrm{H}$, $\mathrm{C}_{6} \mathrm{H}_{5}$, pyridine $\left.3 \mathrm{H}\right)$; MS: $\mathrm{m} / z 295\left(\mathrm{M}^{+}, 65 \%\right)$.

Compound (11b): Red crystals from ethanol, yield $77 \%$ (2.26) g, m.p. $140^{\circ}$ C. Calculated for $\mathrm{C}_{15} \mathrm{H}_{15} \mathrm{~N}_{6} \mathrm{O}$ (296.33): C, 61.80; H, 5.44; N, 28.36. Found: C, 60.82; $\mathrm{H}, 5.64 ; \mathrm{N}, 28.49$. IR $\left(\mathrm{U} / \mathrm{cm}^{-1}\right)=3563-3324\left(\mathrm{OH}, \mathrm{NH}_{2}\right)$, $3053\left(\mathrm{CH}\right.$ aromatic), $2980\left(\mathrm{CH}_{3}\right), 1657(\mathrm{C}=\mathrm{N}), 1637(\mathrm{C}$ $=\mathrm{C}) .{ }^{1} \mathrm{HNMR}(\delta \mathrm{ppm}): 2.82\left(\mathrm{~s}, 3 \mathrm{H}, \mathrm{CH}_{3}\right), 4.32(\mathrm{~s}, 2 \mathrm{H}$, $\mathrm{D}_{2} \mathrm{O}$ exchangeable, $\left.\mathrm{NH}_{2}\right), 5.18\left(\mathrm{~s}, 2 \mathrm{H}\right.$, triazine $\left.\mathrm{CH}_{2}\right), 7.16$ $7.39\left(\mathrm{~m}, 8 \mathrm{H}, \mathrm{C}_{6} \mathrm{H}_{5}\right.$, pyridine $\left.3 \mathrm{~h}\right), 10.21(\mathrm{~s}, 1 \mathrm{H}, \mathrm{OH})$; MS: $\mathrm{m} / \mathrm{z} 296\left(\mathrm{M}^{+}, 30 \%\right)$.

2-(2-Phenylhydrazono)-2-(5-methyl-6H-1,3,4-oxadi azin-2-yl)acetonitrile (13a) and 2-(2-p-chlorolhydrazono)-2-(5-methyl-6H-1,3,4-oxadiazin-2-yl)acetonitril e (13b).

General procedure: To a cold solution $\left(0^{\circ} \mathrm{C}-5^{\circ} \mathrm{C}\right)$ of 3 $(1.73 \mathrm{~g}, 0.01 \mathrm{~mol})$ in ethanol $(30 \mathrm{~mL})$ containing sodium hydroxide $(10 \%, 6 \mathrm{~mL})$ either benzenediazonium chloride (7) $(0.01 \mathrm{~mol})$ or P-chloro benzenediazonium chloride (12) obtained via the addition of sodium nitrite solution $(0.70 \mathrm{~g}, 0.01 \mathrm{~mol})$ to a cold solution $\left(0^{\circ} \mathrm{C}-5^{\circ} \mathrm{C}\right)$ of aniline or p-chloro aniline $(1.27 \mathrm{~g}, 0.01 \mathrm{~mol})(0.01 \mathrm{~mol})$ $(0.93 \mathrm{~g}, 0.01 \mathrm{~mol})$ in hydrochloric acid $(8 \mathrm{~mL})$ was added with continuous stirring was added with continuous stirring. The reaction mixture was stirred for an additional 2 $\mathrm{h}$ then the formed solid product was collected by filtration.

Compound 13a: Red crystals from ethanol, yield, $71 \%$ (1.71) g, m.p. $236^{\circ} \mathrm{C}-240^{\circ} \mathrm{C}$. Calculated for $\mathrm{C}_{12} \mathrm{H}_{11} \mathrm{~N}_{5} \mathrm{O}$ (241.23): C, $59.74 ; \mathrm{H}, 4.59 ; \mathrm{N}, 29.03$. Found: C, 60.01; H, 4.93; N, 28.7 0. IR $\left(\mathrm{U} / \mathrm{cm}^{-1}\right)=3467-3322$ $(\mathrm{NH}), 3055\left(\mathrm{CH}\right.$ aromatic), $2974\left(\mathrm{CH}_{3}\right), 2227(\mathrm{CN})$, $1656(\mathrm{C}=\mathrm{N}), 1637(\mathrm{C}=\mathrm{C}) .{ }^{1} \mathrm{HNMR}(\delta \mathrm{ppm}): 2.66(\mathrm{~s}, 3 \mathrm{H}$, $\left.\mathrm{CH}_{3}\right), 5.26\left(\mathrm{~s}, 2 \mathrm{H}\right.$, triazine $\left.\mathrm{CH}_{2}\right), 7.23-7.36(\mathrm{~m}, 5 \mathrm{H}$, $\left.\mathrm{C}_{6} \mathrm{H}_{5}\right), 8.26$ (s, $\left.1 \mathrm{H}, \mathrm{NH}\right)$; MS: $m / z 241\left(\mathrm{M}^{+}, 70 \%\right)$.

Compound 13b: Red crystals from ethanol, yield, $70 \%\left(1.92\right.$ g), m.p. $100^{\circ}$ C. Calculated for $\mathrm{C}_{12} \mathrm{H}_{10} \mathrm{~N}_{5} \mathrm{OCl}$ (275.68): Calcd: C, 52.28; H, 3. 66; N, 25. 40. Found: C, 51.97; H, 3.45; N, 25.23. IR $\left(\mathrm{U} / \mathrm{cm}^{-1}\right)=3573-3312$ $(\mathrm{NH}), 3053(\mathrm{CH}$ aromatic $), 2983\left(\mathrm{CH}_{3}\right), 1650(\mathrm{C}=\mathrm{N})$, $1634(\mathrm{C}=\mathrm{C}) .{ }^{1} \mathrm{HNMR}(\delta \mathrm{ppm}): 2.80\left(\mathrm{~s}, 3 \mathrm{H}, \mathrm{CH}_{3}\right), 5.16(\mathrm{~s}$, $2 \mathrm{H}$, triazine $\left.\mathrm{CH}_{2}\right), 7.28-7.37\left(\mathrm{~m}, 4 \mathrm{H}, \mathrm{C}_{6} \mathrm{H}_{4}\right), 8.24(\mathrm{~s}, 1 \mathrm{H}$, 
$\mathrm{NH})$; MS: $m / z 275\left(\mathrm{M}^{+}, 80 \%\right)$.

3-(5-Amino-3-methyl-1H-pyrazol-1-yl)-3-oxo-propa nenitrile (15).

To a solution compound $3(1.73 \mathrm{~g}, 0.01 \mathrm{~mol})$ in ethanol $(25 \mathrm{~mL})$ in a water bath at $60^{\circ} \mathrm{C}$ potassium cyanide (14) $(0.65 \mathrm{~g}, 0.01 \mathrm{~mol})$ was added with continues stirring. The reaction mixture was left in water bath for $30 \mathrm{~min}$ at $60^{\circ} \mathrm{C}$ then poured onto a beaker containing ice/water mixture and drops of hydrochloric acid. The formed solid product was collected by filtration and dried. Orange crystals from ethanol in a yield $59 \%(0.96 \mathrm{~g})$, with m.p. $148^{\circ} \mathrm{C}-150^{\circ} \mathrm{C}$. Calculated for $\mathrm{C}_{7} \mathrm{H}_{8} \mathrm{~N}_{4} \mathrm{O}$ (164.15): $\mathrm{C}$, 51.21; H, 4.90; N, 34.12. Found : C, 51.41; H, 4.82; N, 34.10. IR $\left(\mathrm{U} / \mathrm{cm}^{-1)}=3468-3348\left(\mathrm{NH}_{2}\right), 3055(\mathrm{CH}\right.$ aromatic), $2984\left(\mathrm{CH}_{3}\right), 2227(\mathrm{CN}), 1644(\mathrm{C}=\mathrm{N}), 1633$ $(\mathrm{C}=\mathrm{C}) .{ }^{1} \mathrm{HNMR}(\delta \mathrm{ppm}): 2.74\left(\mathrm{~s}, 3 \mathrm{H}, \mathrm{CH}_{3}\right), 4.11(\mathrm{~s}, 2 \mathrm{H}$, $\mathrm{CH}_{2}$ ), 4.84 (s, $2 \mathrm{H}, \mathrm{NH}_{2}$ ), 5.53 (s, $1 \mathrm{H}$, pyrazole); MS: $m / z$ $164\left(\mathrm{M}^{+}, 33 \%\right)$.

2-(5-Methyl-6H-[1,3,4]oxadiazin-2-yl)-3-phenyl-acr ylonitrile (18)

Equimolecular amounts of $3(1.73 \mathrm{~g}, 0.01 \mathrm{~mol})$ and benzaldehyde (16) (1.06 g, $0.01 \mathrm{~mol})$ in 1,4-dioxane (30 $\mathrm{mL})$ containing piperidine $(1.00 \mathrm{~mL})$ were heated under reflux for $3 \mathrm{~h}$. The solid product formed upon dilution with ice/water containing hydrochloric acid $\left(\right.$ till $\left.\mathrm{pH}_{6}\right)$, was collected by filtration. Red crystals from ethanol in a yield $76 \%(1.71 \mathrm{~g})$ with m.p. $100^{\circ} \mathrm{C}$. Calculated for $\mathrm{C}_{13} \mathrm{H}_{11} \mathrm{~N}_{3} \mathrm{O}$ (225.24): C, 69.32; H, 4.92; N,18.66. Found: C, 68.84; H, 5.23; N,18.55. IR $\left(\mathrm{V} / \mathrm{cm}^{-1}\right)=3057(\mathrm{CH}$ aromatic), $2985\left(\mathrm{CH}_{3}\right), 2234(\mathrm{CN}), 1641(\mathrm{C}=\mathrm{N}), 1633$ $(\mathrm{C}=\mathrm{C}) .{ }^{1} \mathrm{HNMR}(\delta \mathrm{ppm}): 2.68\left(\mathrm{~s}, 3 \mathrm{H}, \mathrm{CH}_{3}\right), 5.26(\mathrm{~s}, 2 \mathrm{H}$, oxadiazine- $\left.\mathrm{CH}_{2}\right), 6.05(\mathrm{~s}, 1 \mathrm{H}, \mathrm{CH}=\mathrm{C}), 7.22-7.35(\mathrm{~m}, 5 \mathrm{H}$, $\left.\mathrm{C}_{6} \mathrm{H}_{5}\right) ; \mathrm{MS}: m / z 255\left(\mathrm{M}^{+}, 80 \%\right)$.

6-Amino-2,8-dihydro-3-methyl-8-phenylpyrido[2,1b][1,3,4]oxadiazine-7,9-dicarbonitrile (20a) and 2,8Di-hydro-6-hydroxy-3-methyl-8-phenylpyrido[2,1-b][ 1,3,4] ]oxadiazine-7,9-dicarbonitrile (20b).

General procedure: Equimolecular amounts of $\mathbf{1 8}$ $(2.25 \mathrm{~g}, 0.01 \mathrm{~mol})$ either malononitrile (10a) $(0.66 \mathrm{~g}$, $0.01 \mathrm{~mol})$ or ethyl cyanoactate (10b) were added(1.13 g, $0.01 \mathrm{~mol})$ in 1,4-dioxane $(25 \mathrm{~mL})$ containing triethylamine $(1.00 \mathrm{~mL})$ were heated under reflux for $4 \mathrm{~h}$. The solid product formed upon dilution with ice/water containing hydrochloric acid (till $\mathrm{pH}_{6}$ ), was collected by filtration.

Compound (20a): Brown crystals from ethanol, yield $71 \%\left(2.06\right.$ g), m.p. $110^{\circ}$ C. Calculated for $\mathrm{C}_{16} \mathrm{H}_{13} \mathrm{~N}_{5} \mathrm{O}$ (291.26): C, 65.97; H, 4.50; N, 24.04. Found: C, 65.79; $\mathrm{H}, 4.36 ; \mathrm{N}, 23.98$. IR $\left(\mathrm{U} / \mathrm{cm}^{-1}\right)=3473-3344\left(\mathrm{NH}_{2}\right)$, $3057\left(\mathrm{CH}\right.$ aromatic), $2980\left(\mathrm{CH}_{3}\right), 2222,2220(2 \mathrm{CN})$, $1662(\mathrm{C}=\mathrm{N}), 1640(\mathrm{C}=\mathrm{C}) .{ }^{1} \mathrm{HNMR}(\delta \mathrm{ppm}): 2.79(\mathrm{~s}, 3 \mathrm{H}$, $\left.\mathrm{CH}_{3}\right), 4.43\left(1 \mathrm{~s}, 2 \mathrm{H}, \mathrm{D}_{2} \mathrm{O}\right.$ exchangeable, $\left.\mathrm{NH}_{2}\right), 5.20(\mathrm{~s}, 2 \mathrm{H}$, Oxadiazine $\mathrm{CH}_{2}$ ), $5.52(\mathrm{~s}, 1 \mathrm{H}$, pyridine $\mathrm{H}-4), 7.23$ - 7.36 (m, 5H, $\left.\mathrm{C}_{6} \mathrm{H}_{5}\right)$; MS: $m / z 291\left(\mathrm{M}^{+}, 40 \%\right)$.

Compound (20b): Orange crystals from 1,4-dioxane, yield $69 \%$, (2 g), m.p. $80^{\circ}$ C. Calculated for $\mathrm{C}_{16} \mathrm{H}_{12} \mathrm{~N}_{4} \mathrm{O}_{2}$ (292.25): C, 65.75; H, 4.13; N,19.16. Found: C, 66.10; H, 4.43; N,19.58. IR $\left(U / \mathrm{cm}^{-1}\right)=3852-3480(\mathrm{OH}), 2980$ $\left(\mathrm{CH}_{3}\right), 2228,2218(2 \mathrm{CN}), 1644(\mathrm{C}=\mathrm{N}), 1636(\mathrm{C}=\mathrm{C})$. ${ }^{1} \mathrm{HNMR}(\delta \mathrm{ppm}): 2.78\left(\mathrm{~s}, 3 \mathrm{H}, \mathrm{CH}_{3}\right), 5.21(\mathrm{~s}, 2 \mathrm{H}$, oxadiazine- $\left.\mathrm{CH}_{2}\right), 5.51(\mathrm{~s}, 1 \mathrm{H}$, pyridine $\mathrm{H}-4), 7.21-7.33(\mathrm{~m}$, $\left.5 \mathrm{H}, \mathrm{C}_{6} \mathrm{H}_{5}\right), 10.22\left(\mathrm{~s}, 1 \mathrm{H}, \mathrm{D}_{2} \mathrm{O}\right.$ exchangeable, $\left.\mathrm{OH}\right) ; \mathrm{MS}$ : $\mathrm{m} / \mathrm{z} 292\left(\mathrm{M}^{+}, 32 \%\right)$.

N'-(2-bromocyclohexylidene)-2-cyanoacetohydrazi de (22).

To a hot solution of cyanoacetylhydrazine (1) $(0.99 \mathrm{~g}$, $0.01 \mathrm{~mol})$, in 1,4-dioxane $(40 \mathrm{~mL}), \alpha$-bromocyclohexanone (21) (1.76 g, $0.01 \mathrm{~mol})$ was added and drops of piperidine $(1 \mathrm{~mL})$. The reaction mixture was kept at room temperature with stirring for $1 \mathrm{~h}$ and the formed solid product was filtrated off. Orange crystals from 1,4-dioxane in a yield $88 \%(2.27 \mathrm{~g})$ with m.p. $137^{\circ} \mathrm{C}-$ $140^{\circ} \mathrm{C}$. Calculated for $\mathrm{C}_{9} \mathrm{H}_{12} \mathrm{BrN}_{3} \mathrm{O}$ (258.12): $\mathrm{C}, 41.88 ; \mathrm{H}$, 4.69; Br, 30.96; N, 16.27. Found: C, 41.67; H, 4.33; Br, $31.05 ; \mathrm{N}, 16.34$. IR $\left(\mathrm{V} / \mathrm{cm}^{-1}\right)=3466-3329(\mathrm{NH}), 3055$ $(\mathrm{CH}$ aromatic), $2260(\mathrm{CN}), 1640(\mathrm{C}=\mathrm{N}), 1683(\mathrm{C}=\mathrm{O})$, $1630(\mathrm{C}=\mathrm{C}) .{ }^{1} \mathrm{HNMR}(\delta \mathrm{ppm}): 2.21-2.27\left(\mathrm{~m}, 8 \mathrm{H}, 4 \mathrm{CH}_{2}\right)$, $3.88\left(\mathrm{~s}, 2 \mathrm{H}, \mathrm{CH}_{2}\right), 4.22(\mathrm{~s}, 1 \mathrm{H}, \mathrm{CH}), 8.30\left(\mathrm{~s}, 1 \mathrm{H}, \mathrm{D}_{2} \mathrm{O}\right.$ exchangeable, NH); MS: $m / z 258\left(\mathrm{M}^{+}, 90 \%\right)$.

1-amino-5,6,7,8-tetrahydro-4aHbenzo[e]pyrazolo[5, 1-c][1,2,4]triazine (24a) and 1-Phenyl-5,6,7,8-tetrahydro-4aHbenzo [e]pyrazolo[5,1-c][1,2,4]triazine (24b).

A mixture of $22(2.588 \mathrm{~g}, 0.01 \mathrm{~mol})$ and hydrazine hydrate (4a) $(0.99 \mathrm{~g}, 0.01 \mathrm{~mol})$ was heated in an oil bath $140^{\circ} \mathrm{C}$ for $1 \mathrm{~h}$, then left to cool. The remaining product was heated in ethanol then poured into ice/water mixture and the formed solid product was collected by filtration.

Compound (24a): Brown crystals from 1,4-dioxane, yield $74 \%\left(1.41\right.$ g), m.p. $>300^{\circ} \mathrm{C}$. Calculated for $\mathrm{C}_{9} \mathrm{H}_{13} \mathrm{~N}_{5}$ (191.23): C, 56.52; H, 6.85; N, 36.62. Found: $\mathrm{C}$, 56.85; H, 6.60; N, 36.98. IR $\left(\mathrm{U} / \mathrm{cm}^{-1}\right)=3478-3321$ $\left(\mathrm{NH}_{2}, \mathrm{NH}\right), 3058(\mathrm{CH}$ aromatic $), 1643(\mathrm{C}=\mathrm{N}), 1636(\mathrm{C}=$ C). ${ }^{1} \mathrm{HNMR}(\delta \mathrm{ppm}): 2.20-2.25\left(\mathrm{~m}, 4 \mathrm{H}, 2 \mathrm{CH}_{2}\right), 2.33-$ $2.41\left(\mathrm{~m}, 5 \mathrm{H}, 2 \mathrm{CH}_{2}, \mathrm{CH}\right), 4.82\left(\mathrm{~s}, 2 \mathrm{H}, \mathrm{D}_{2} \mathrm{O}\right.$ exchangeable, $\left.\mathrm{NH}_{2}\right), 5.32(\mathrm{~s}, 1 \mathrm{H}$, pyrazole $\mathrm{H}-4), 8.25\left(\mathrm{~s}, 1 \mathrm{H}, \mathrm{D}_{2} \mathrm{O}\right.$ exchangeable, NH); MS: $m / z 191\left(\mathrm{M}^{+}, 100 \%\right)$.

Compound (24b): Equimolecular amounts of 22 (2.58 $\mathrm{g}, 0.01 \mathrm{~mol})$ and phenylhydrazine (4b) $(1.08 \mathrm{~g}, 0.01 \mathrm{~mol})$ in 1,4-dioxane $(40 \mathrm{~mL})$ were heated under reflux for $4 \mathrm{~h}$. The solid product formed upon dilution with ice/water containing hydrochloric acid (till $\mathrm{pH}_{6}$ ), was collected by filtration.

Compound (24b): Brown crystals from 1,4- dioxane, yield $78 \%\left(2.08\right.$ g), m.p. $217^{\circ} \mathrm{C}-220^{\circ} \mathrm{C}$. Calculated for $\mathrm{C}_{15} \mathrm{H}_{17} \mathrm{~N}_{5}$ (267.31): C, 67.39; H, 6.41; N, 26.19. Found: C, 67.69; H,6.32; N, 26.74. IR $\left(\mathrm{V} / \mathrm{cm}^{-1}\right)=3465-3330$ $\left(\mathrm{NH}_{2}\right), 3056(\mathrm{CH}$ aromatic), $1640(\mathrm{C}=\mathrm{N}), 1634(\mathrm{C}=\mathrm{C})$. ${ }^{1} \mathrm{H}$ NMR $(\delta \mathrm{ppm}): 2.23-2.28\left(\mathrm{~m}, 4 \mathrm{H}, 2 \mathrm{CH}_{2}\right), 2.32-2.39$ $\left(\mathrm{m}, 5 \mathrm{H}, 2 \mathrm{CH}_{2}, \mathrm{CH}\right), 4.80\left(\mathrm{~s}, 2 \mathrm{H}, \mathrm{D}_{2} \mathrm{O}\right.$ exchangeable, $\left.\mathrm{NH}_{2}\right), 5.38(\mathrm{~s}, 1 \mathrm{H}$, pyrazole $\mathrm{H}-4), 7.28-7.36(\mathrm{~m}, 5 \mathrm{H}$, 
$\left.\mathrm{C}_{6} \mathrm{H}_{5}\right) ; \mathrm{MS}: m / z 267\left(\mathrm{M}^{+}, 100 \%\right)$.

2-(2-Phenylhydrazono)-2-(5,6,7,8-tetrahydro-4aH-b enzo[e][1,3,4]oxadiazin-3-yl)acetonitrile (25a) and 2(2-4-chlorophenylhydrazono)-2-(5,6,7,8-tetrahydro-4a $H$-benzo[e][1,3,4]oxadiazin-3-yl)acetonitrile (25b).

General procedure: To a cold solution $\left(0^{\circ} \mathrm{C}-5^{\circ} \mathrm{C}\right)$ of $22(2.58 \mathrm{~g}, 0.01 \mathrm{~mol})$ in ethanol $(35 \mathrm{~mL})$ containing sodium hydroxide $(6 \mathrm{~mL}, 10 \%)$ and a solution of benzenediazonium chloride $(0.01 \mathrm{~mol})$ or p-chlorobenzenediazonium chloride $(0.01 \mathrm{~mol})$ obtained via the addition of sodium nitrite solution $(0.70 \mathrm{~g}, 0.01 \mathrm{~mol})$ to a cold solution $\left(0^{\circ} \mathrm{C}-5^{\circ} \mathrm{C}\right)$ of either aniline $(0.93 \mathrm{~g}, 0.01 \mathrm{~mol})$ or p-chloroaniline $(1.27 \mathrm{~g}, 0.01 \mathrm{~mol})$ containing the appropriate amounts of hydrochloric acid $(10 \mathrm{~mL})$ was added with continuous stirring] was added with continuous stirring. The reaction mixture was stirred for an additional $2 \mathrm{~h}$ then the formed solid product was collected by filtration.

Compound (25a): Red crystals from ethanol, yield, $82 \%\left(2.31\right.$ g), m.p. $110^{\circ} \mathrm{C}$. Calculated for $\mathrm{C}_{15} \mathrm{H}_{15} \mathrm{~N}_{5} \mathrm{O}$ (281.30): C, 64.04; H, 5.37; N, 24.89. Found: C, 64.44; $\mathrm{H}, 5.00 ; \mathrm{N}, 24.66$. IR $\left(\mathrm{V} / \mathrm{cm}^{-1}\right)=3466-3316(\mathrm{NH}), 3053$ $\left(\mathrm{CH}\right.$ aromatic), $2890\left(\mathrm{CH}_{2}\right), 2246(\mathrm{CN}), 1646(\mathrm{C}=\mathrm{N})$, $1632(\mathrm{C}=\mathrm{C}) .{ }^{1} \mathrm{HNMR}(\delta \mathrm{ppm}): 2.22-2.27\left(\mathrm{~m}, 4 \mathrm{H}, 2 \mathrm{CH}_{2}\right)$, $2.30-2.43\left(\mathrm{~m}, 5 \mathrm{H}, 2 \mathrm{CH}_{2}, \mathrm{CH}\right), 7.26-7.38(\mathrm{~m}, 5 \mathrm{H}$, $\left.\mathrm{C}_{6} \mathrm{H}_{5}\right), 8.28$ (s, $\left.1 \mathrm{H}, \mathrm{NH}\right)$; MS: $m / z 281\left(\mathrm{M}^{+}, 20 \%\right)$.

Compound (25b): Orange crystals from ethanol, yield $85 \%\left(2.72\right.$ g), m.p. $120^{\circ}$ C. Calculated for $\mathrm{C}_{15} \mathrm{H}_{14} \mathrm{~N}_{5} \mathrm{OCl}$ (315.74): C, 57.06; H, 4.47; Cl, 11.23; N, 22.18. Found: C, 57.45; H, 4.52; Cl, 11.09; N, 21.98. $\operatorname{IR}\left(U / \mathrm{cm}^{-1}\right)=$ $3475-3322(\mathrm{NH}), 3059(\mathrm{CH}$ aromatic), $2251(\mathrm{CN}), 1649$ $(\mathrm{C}=\mathrm{N}), 1630(\mathrm{C}=\mathrm{C}) .{ }^{1} \mathrm{HNMR}(\delta \mathrm{ppm}): 2.25-2.29(\mathrm{~m}$, $\left.4 \mathrm{H}, 2 \mathrm{CH}_{2}\right), 2.34-2.40\left(\mathrm{~m}, 5 \mathrm{H}, 2 \mathrm{CH}_{2}, \mathrm{CH}\right), 7.29-7.42$ (m, 4H, $\left.\mathrm{C}_{6} \mathrm{H}_{4}\right), 8.24(\mathrm{~s}, 1 \mathrm{H}, \mathrm{NH})$; MS: $m / z 315\left(\mathrm{M}^{+}, 70 \%\right)$.

\section{Conclusion}

The present work describes the synthesis of the new hydrazide-hydrazone derivative 3 . The latter was used for the synthesis of a series of heterocyclic products with antitumor activities. Compounds 11a and 20b showed the highest inhibitor effect towards the tested three cancer cells. The work opened the door towards pharmaceutical uses of other hydrazide-hydrazones that could be obtained by the sequence described in this article.

\section{Acknowledgements}

R. M. Mohareb thanks the Alexander von Humboldt Foundation for the financial support during summer, 2009 during his fellowship in Germany (Erlangen) for doing research and completing this work.

\section{REFERENCES}

[1] C. Loncle, J. M. Brunel, N. Vidal, M. Dherbomez and Y.
Letourneux, "Synthesis and Antifungal Activity of Cholesterol-Hydrazone Derivatives," European Journal of Medicinal Chemistry, Vol. 39, No. 12, 2004, pp. 10671071. doi:10.1016/j.ejmech.2004.07.005

[2] S. P. Garoufalias, N. Pouli, P. A. Marakos and G. Ladas, "Synthesis Antimicrobial and Antifungal Activity of Some New 3-Substituted Derivatives of 4-(2,4-Dichlorophenyl)5-Adamantyl-1 H-1,2,4-Triazole," II Farmaco, 2002, Vol. 57, No. 12, pp. 973-977.

doi:10.1016/S0014-827X(02)01227-2

[3] P. Vicini, F. Zani, P. Cozzini and I. Doytchinova, "Hydrazones of 1,2-Benzisothiazole Hydrazides: Synthesis, Antimicrobial Activity and QSAR Investigations," European Journal of Medicinal Chemistry, Vol. 37, No. 7, 2002, pp. 553-564. doi:10.1016/S0223-5234(02)01378-8

[4] F. D. Popp, "Potential Anticonvulsant XII. Anticonvulsant Activity of Some Aldehyd Derivatives," European Journal of Medicinal Chemistry, Vol. 24, No. 3, 1989, pp. 313-315. doi:10.1016/0223-5234(89)90016-0

[5] S. K. Sridhar, S. N. Pandeya, J. P. Stables and A. Ramesh, "Anti-Convulsant Activity of Hydrazones, Schiff and Mann Basis of Istatin Derivatives," European Journal of Pharmaceutical Science, Vol. 16, No. 3, 2002, pp. 129132. doi:1016/S0928-0987(02)00077-5

[6] S. G. Küçükgüzel, A. Mazi, F. Sahin, S. Öztürk and J. P. Stables, "Synthesis and Biological Activities of Diflunisal Hydrazide-Hydrazones," European Journal of Medicinal Chemistry, Vol. 38, No. 11, 2003, pp. 1005-1013. doi:10.1016/j.ejmech.2003.08.004

[7] M. A. Gaston, L. R. Dias, A. C. Freitas, A. L. Miranda and P. Barreiro, "Synthesis and Analgesic Properties of New 4-Arylhydrazone 1-H Pyrazole [3,4-b] Pyridine Derivatives," Pharmaceutica Acta Helvetiae, Vol. 71, No. 2, 1996, pp. 213-219. doi:10.1016/0031-6865(96)00012-X

[8] S. Gemma, G. Kukreja, C. Fattorusso, M. Persico, M. Romano, M. Altarelli, L. Savini, G. Campiani, E. Fattorusso and N. Basilico, "Synthesis of N1-Arylidene-N2Quinolyl- and N2-Acrydinylhydrazones as Potent Antimalarial Agents Active against CQ-Resistant P. falciparumstrains," Bioorganic and Medicinal Chemistry Letters, Vol. 16, No. 20, 2006, pp. 5384-5388. doi:10.1016/j.bmcl.2006.07.060

[9] A. D. Pilla, S. Rani, P. D. Rathod, F. P. Xanvier, K. Vasu, K. H. Padh and V. Sudarsanam, "QSAR Studies on Some Thiophene Analogs as Anti-Inflammatory Agents: Enhancement of Activity by Electronic Parameters and Its Utilization for Chemical Lead Optimization," Bioorganic and Medicinal Chemistry, Vol. 13, No. 4, 2005, pp. 12751283. doi:10.1016/j.bmc.2004.11.016

[10] P. Melnyk, V. Leroux, C. Sergheraert and P. Grellier, "Design, Synthesis and in Vitro Antimalarial Activity of an Acylhydrazone Library," Bioorganic and Medicinal Chemistry Letters, Vol. 16, No. 1, 2006, pp. 31-35. doi:10.1016/j.bmcl.2005.09.058

[11] S. G. Küçükgüzel, S. Rollas, I. Küçükgüzel and M. Kiraz, "Synthesis and Antimycobacterial Activity of Some Coupling Products from 4-Aminobenzoic Acid Hydrazones," European Journal of Medicinal Chemistry, Vol. 34, No. 12, 1999, pp. 1093-1100. 
doi:10.1016/S0223-5234(99)00129-4

[12] J. Patole, U. Sandbhor, S. Padhye, D. N. Deobagkar, C. Anson and A. E. Powell, "Structural Chemistry and in Vitro Antitubercular Activity of Acetylpyridine Benzoyl Hydrazone and Its Copper Complex against Mycobacterium smegmatis," Bioorganic and Medicinal Chemistry Letters, Vol. 13, No. 1, 2003, pp. 51-55. doi:10.1016/S0960-894X(02)00855-7

[13] R. Maccari, R. Ottana and M. G. Vigorita, "In Vitro Advanced Antimycobacterial Screening of Isoniazid-Related Hydrazones, Hydrazides and Cyanoboranes: Part 14," Bioorganic and Medicinal Chemistry Letters, Vol. 15, No. 10, 2005, pp. 2509-2513. doi:10.1016/j.bmcl.2005.03.065

[14] M. T. Cocco, C. Congiu, V. Onnis, M. C. Pusceddu, M. L. Schivo, A. Logu, E. Gürsoy and N. U. Güzeldemirci, "Synthesis and Primary Cytotoxicity Evaluation of New Imidazo[2,1-b]Thiazole Derivatives," European Journal of Medicinal Chemistry, Vol. 42, No. 3, 2007, pp. 320326. doi:10.1016/j.ejmech.2006.10.012

[15] N. Karal, A. Kocabalkanl, A. Gürsoy and O. Ateş, "Syn- thesis and Antitubercular Activity of 4-(3-Coumarinyl)3-Cyclohexyl-4-Thiazolin-2-One Benzylidenehydrazon," II Farmaco, Vol. 57, No. 7, 2002, pp. 589-593. doi:10.1016/S0014-827X(02)01254-5

[16] V. Suni, M. R. Kurup and M. Nethaji, "Unusual Isolation of a Hemiaminal Product from 4-Cyclohexyl-3-Thiosemicarbazide and Di-2-Pyridyl Ketone: Structural and Spectral Investigations," Journal of Molecular Structure, Vol. 749, No. 1-3, 2005, pp. 177-182. doi:10.1016/j.ejmech.2006.10.012

[17] G. Kuecuekguezel, A. Kocatepe, E. D. Clercq, F. Şahin and M. Guelluece, "Synthesis and Biological Activity of 4-Thiazolidinones, Thiosemicarbazides Derived from Diflunisal Hydrazide," Cheminform, Vol. 37, No. 34, 2006, pp. 353-359. doi:10.1002/chin.200634124

[18] A. P. Hoener, H. B. Frutos and J. C. Gauvin, "Novel OnePot Microwave Assisted Gewald Synthesis of 2-Acylamino Thiophenes on Solid Support," Synlett, Vol. 1, No. 1, 2003, pp. 63-67. doi:10.1055/s-2003-36229 